\title{
Suppression of phase mixing in drift-kinetic plasma turbulence
}

J. T. Parker', E. G. Highcock, A. A. Schekochihin, and P. J. Dellar

Citation: Physics of Plasmas 23, 070703 (2016); doi: 10.1063/1.4958954

View online: http://dx.doi.org/10.1063/1.4958954

View Table of Contents: http://aip.scitation.org/toc/php/23/7

Published by the American Institute of Physics

\section{Articles you may be interested in}

Electron holes in phase space: What they are and why they matter

Physics of Plasmas 24, 055601 (2017); 10.1063/1.4976854

Radiation pressure acceleration of protons to $93 \mathrm{MeV}$ with circularly polarized petawatt laser pulses Physics of Plasmas 23, 070701 (2016); 10.1063/1.4958654

Magnetohydrodynamics for collisionless plasmas from the gyrokinetic perspective

Physics of Plasmas 23, 070705 (2016); 10.1063/1.4960029

Two-fluid biasing simulations of the large plasma device

Physics of Plasmas 24, 022303 (2017); 10.1063/1.4975616

Characterizing fluid and kinetic instabilities using field-particle correlations on single-point time series Physics of Plasmas 24, 055901 (2017); 10.1063/1.4977465

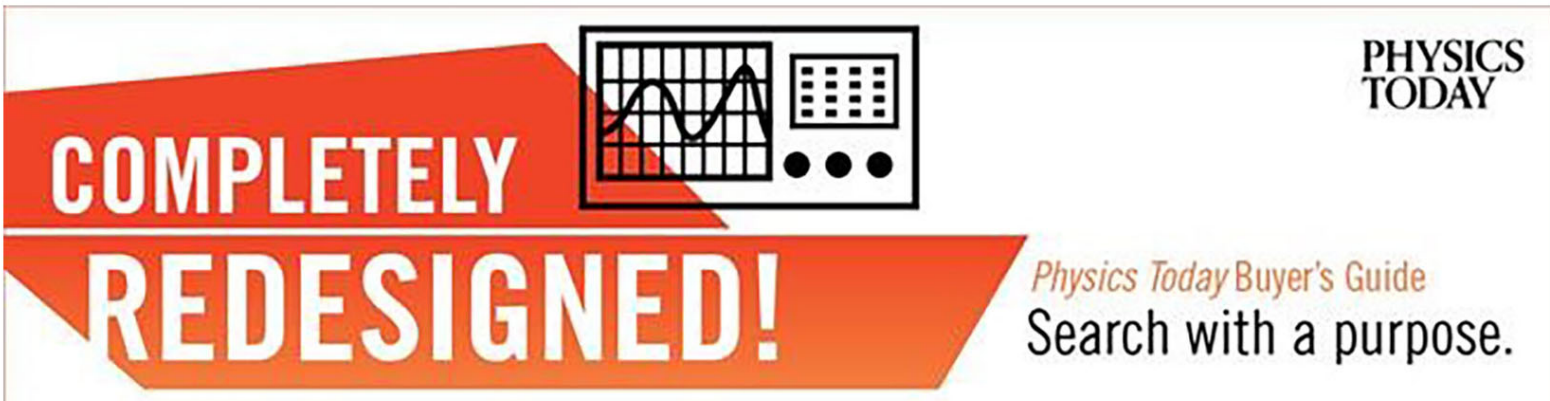




\title{
Suppression of phase mixing in drift-kinetic plasma turbulence
}

\author{
J. T. Parker, ${ }^{1,2,3, a)}$ E. G. Highcock, ${ }^{3,4}$ A. A. Schekochihin,, ${ }^{4,5}$ and P. J. Dellar ${ }^{2}$ \\ ${ }^{1}$ Science and Technology Facilities Council, Rutherford Appleton Laboratory, Harwell Campus, \\ Didcot OX11 0QX, United Kingdom \\ ${ }^{2}$ OCIAM, Mathematical Institute, University of Oxford, Andrew Wiles Building, \\ Radcliffe Observatory Quarter, Woodstock Road, Oxford OX2 6GG, United Kingdom \\ ${ }^{3}$ Brasenose College, Radcliffe Square, Oxford OX1 4AJ, United Kingdom \\ ${ }^{4}$ Rudolf Peierls Centre for Theoretical Physics, University of Oxford, 1 Keble Road, Oxford OXI 3NP, \\ United Kingdom \\ ${ }^{5}$ Merton College, Merton Street, Oxford OXI 4JD, United Kingdom
}

(Received 18 May 2016; accepted 5 July 2016; published online 15 July 2016)

Transfer of free energy from large to small velocity-space scales by phase mixing leads to Landau damping in a linear plasma. In a turbulent drift-kinetic plasma, this transfer is statistically nearly canceled by an inverse transfer from small to large velocity-space scales due to "anti-phasemixing" modes excited by a stochastic form of plasma echo. Fluid moments (density, velocity, and temperature) are thus approximately energetically isolated from the higher moments of the distribution function, so phase mixing is ineffective as a dissipation mechanism when the plasma collisionality is small. Published by AIP Publishing. [http://dx.doi.org/10.1063/1.4958954]

Kinetic turbulence in weakly collisional, strongly magnetized plasmas is ubiquitous in magnetic-confinement-fusion experiments ${ }^{1-3}$ and in astrophysical settings. ${ }^{4,5}$ Like fluid turbulence, kinetic turbulence may be described as the injection (e.g., by a plasma instability), cascade to small scales, and dissipation of a quadratic invariant, viz., free energy. On spatial scales larger than the ion Larmor radius, kinetic turbulence incorporates two mechanisms for dissipating free energy into heat. The first is a fluid-like nonlinear cascade from large to smaller, sub-Larmor, spatial scales (where the free energy is dissipated eventually by collisions ${ }^{5-8}$ ). The second is parallel phase mixing, a linear process that transfers free energy from the fluid moments (density, fluid velocity, and temperature) to the kinetic (higher-order) moments by creating perturbations in the velocity distribution on ever finer scales in velocity space, perturbations which are also then dissipated by collisions. In a linear plasma, this is known as Landau damping and the free energy is dissipated at a rate independent of collision frequency. ${ }^{9,10}$

The macroscopic properties of the turbulence (such as heat and momentum transport) are directly affected by the two energy dissipation channels; yet, while each is understood in isolation, how they interact is not clear. Recent work leads to some disquieting observations. First, a fluidlike theory for the nonlinear cascade ${ }^{11}$ predicts power-law spectra for the electrostatic potential in good agreement with those found in gyrokinetic simulations, but its derivation neglects free-energy transfer by phase mixing, ${ }^{12}$ contrary to what might be expected on the basis of linear theory. Including a constant flux of free energy into velocity space leads to non-universal spectra that tend to be steeper than those empirically observed. ${ }^{12-17}$ Some simulations show a significant proportion of injected free energy cascading and dissipating in velocity space, ${ }^{18}$ albeit with a slower transfer

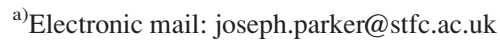

rate than in the linear case, and with a dissipation rate that depends on collision frequency. ${ }^{19,20}$ These observations suggest a complicated relationship between parallel phase mixing and the nonlinear cascade; there is as yet no complete picture of free-energy flow and dissipation in phase space.

In this letter, we propose the outlines of such a picture for electrostatic drift-kinetic turbulence. We show that the net transfer of free energy from fluid to kinetic modes is strongly inhibited in a turbulent plasma, compared to a "linear plasma." This is due to a stochastic version of the classic plasma-echo phenomenon: ${ }^{21,22}$ the nonlinearity excites "anti-phase-mixing" modes that transfer free energy from small to large velocity-space scales, leading to statistical cancellation of the free-energy flux. The significance of this effect depends on the relative rates of phase mixing and nonlinear advection. ${ }^{23,32}$ We identify regions of wavenumber space where either the echo effect dominates or phase mixing occurs at the usual linear rate. Most of the free energy contained in fluid moments is at wavenumbers that lie within the echo-dominated region. Therefore, there is very little net free-energy transfer to fine velocity-space scales via linear phase mixing. Consequently, Landau damping is strongly suppressed as a dissipation mechanism.

We study electrostatic ion-temperature-gradient (ITG) driven drift-kinetic turbulence in an unsheared slab with kinetic ions and Boltzmann electrons. The equations are the drift-kinetic equation for ions

$$
\frac{\partial g}{\partial t}+v_{\|} \nabla_{\|}\left(g+\varphi F_{0}\right)+\boldsymbol{u}_{\perp} \cdot \nabla_{\perp} g=C[g]+\chi,
$$

and the quasineutrality condition ${ }^{24,33,34}$

$$
\varphi \equiv \frac{Z e \phi}{T_{i}}=\alpha \int_{-\infty}^{\infty} \mathrm{d} v_{\|} g, \quad \alpha=\frac{Z T_{e}}{T_{i}} .
$$

Here, $g=\left(1 / n_{i}\right) \int \mathrm{d}^{2} \boldsymbol{v}_{\perp} \delta f$ is the perturbed ion distribution function integrated over perpendicular velocity space, with 
$n_{i}$ the mean ion density, $\phi$ the electrostatic potential, $-e$ the electron charge, $Z e$ the ion charge, and $T_{i}$ and $T_{e}$ the mean ion and electron temperatures; $F_{0}\left(v_{\|} / v_{\text {th }}\right)=e^{-v_{\|}^{2} / v_{\text {th }}^{2}} / \sqrt{\pi}$ is the one-dimensional Maxwellian, with $v_{\|}$the parallel velocity, $v_{\text {th }}=\sqrt{2 T_{i} / m_{i}}$ the ion thermal velocity, and $m_{i}$ the ion mass; $\boldsymbol{u}_{\perp}=\left(\rho_{i} v_{\text {th }} / 2\right) \hat{z} \times \nabla_{\perp} \varphi$ is the $\boldsymbol{E} \times \boldsymbol{B}$ velocity with ion gyroradius $\rho_{i}$, and $\hat{z}$ the unit vector in the direction of the magnetic field line. The perpendicular directions are $x$ and $y$. The energy-injection term due to a mean ITG in the negative $x$ direction is

$$
\chi=-\frac{\rho_{i} v_{\mathrm{th}}}{2 L_{T}} \frac{\partial \varphi}{\partial y}\left(\frac{v_{\|}^{2}}{v_{\mathrm{th}}^{2}}-\frac{1}{2}\right) F_{0}, \quad \frac{1}{L_{T}}=-\frac{\mathrm{d} \ln T_{i}}{\mathrm{~d} x} .
$$

The collision operator $C[g]$ will be described shortly.

The system (1) and (2) conserves the free energy $W=\int \mathrm{d}^{3} \boldsymbol{r} \varphi^{2} / 2 \alpha+\int \mathrm{d}^{3} \boldsymbol{r} \int_{-\infty}^{\infty} \mathrm{d} v_{\|} g^{2} / 2 F_{0}$, except for injection by the ITG and dissipation by collisions

$$
\frac{\mathrm{d} W}{\mathrm{~d} t}=\int \mathrm{d}^{3} \boldsymbol{r} \int_{-\infty}^{\infty} \mathrm{d} v_{\|} \frac{g \chi}{F_{0}}+\int \mathrm{d}^{3} \boldsymbol{r} \int_{-\infty}^{\infty} \mathrm{d} v_{\|} \frac{g C[g]}{F_{0}}
$$

We study the saturated state of drift-kinetic turbulence in a box with parallel and perpendicular lengths $L_{\|}$and $L_{\perp}$. We solve Equations (1) and (2) with SPECTROGK, ${ }^{25,26}$ a phasespace-spectral code designed to capture discrete free-energy conservation exactly. We use a Fourier representation in physical space, $\boldsymbol{r}$, with $128 \times 128 \times 256$ wavenumbers $\boldsymbol{k}$ $=\left(k_{x}, k_{y}, k_{\|}\right)$. We damp the finest resolved scales in physical space by adding a hyperviscous term $-\nu_{h}\left[\left(k_{\perp} / k_{\perp \max }\right)^{8}\right.$ $\left.+\left(k_{\|} / k_{\| \max }\right)^{8}\right] g$ to the right-hand side of (1), with $k_{\perp}$ $=\sqrt{k_{x}^{2}+k_{y}^{2}}$ and $\nu_{h}=1000$, a damping rate chosen to provide sufficient dissipation to absorb the nonlinear cascade without reflection. The exponent 8 concentrates dissipation at the finest scales, allowing an inertial range with a power-law spectrum to emerge even with limited resolution. In the parallel-velocity space, we use the Hermite representation $g\left(v_{\|}\right)=\sum_{m=0}^{\infty} g_{m}$ $H_{m}\left(\hat{v}_{\|}\right) F_{0}\left(\hat{v}_{\|}\right) / \sqrt{2^{m} m !}$, where $H_{m}\left(\hat{v}_{\|}\right)=e^{\hat{v}_{\|}^{2}}\left(-\mathrm{d} / \mathrm{d} \hat{v}_{\|}\right)^{m} e^{-\hat{v}_{\|}^{2}}$ with $\hat{v}_{\|}=v_{\|} / v_{\text {th }}$. For large $m, H_{m} \sim \cos \left(\hat{v}_{\|} \sqrt{2 m}-m \pi / 2\right)$ $\sqrt{2^{m} m !} / F_{0}^{1 / 2}\left(\hat{v}_{\|}\right)$, so $\sqrt{m}$ represents a "wavenumber" in velocity space. The first three Hermite moments are "fluid" quantities: $g_{0}=\varphi / \alpha \quad$ (density), $g_{1}=\sqrt{2} u_{\|} / v_{\text {th }} \quad$ (parallel fluid velocity), and $g_{2}=\delta T_{\|} / T_{i} \sqrt{2}$ (parallel-temperature perturbation). For $m \geq 3, g_{m}$ are "kinetic" moments, representing finer velocity-space scales. We use 256 Hermite modes and model the effects of collisions with 6th-order hypercollisions $C\left[g_{m}\right]=-\nu m^{n} g_{m} \mathcal{I}_{m \geq 3}$, where $n=6, \quad \nu=1000 / 256^{n}$, and $\mathcal{I}_{m \geq 3}=1$ if $m \geq 3$ and $\mathcal{I}_{m \geq 3}=0$ otherwise. For $n=1$, this is Kirkwood's ${ }^{27}$ momentum- and energy-conserving version of the Lenard-Bernstein operator. ${ }^{28}$ The use of hypercollisions in velocity space is analogous to the use of hyperviscosity in physical space and allows us to capture the correct behaviour for linear Landau damping with a much smaller number of Hermite modes. ${ }^{25}$ As the linear growth rate of the ITG instability in drift kinetics increases indefinitely with $k_{y}$, we artificially suppress the temperature gradient by multiplying $\chi$ in (3) by a factor $x=\exp \left[-200\left(k_{\perp}^{2} / k_{\perp \max }^{2}+k_{\|}^{2} / k_{\| \max }^{2}\right)\right]$ to separate the free-energy injection and dissipation scales, as described under (5). This captures the essential feature of ITG turbulence: that the nonlinear turnover rate eventually dominates the injection rate as $k_{\perp}$ increases. However, as the nonlinear and linear characteristic rates $^{11,12}$ are, respectively, $\tau_{\mathrm{nl}}^{-1} \sim k_{\perp}^{4 / 3}$ and $\omega^{*} \sim k_{y}$, it is necessary to limit the free-energy injection artificially to a narrow range of small wavenumbers to allow an inertial range to develop with the available resolution. Then only low wavenumbers can grow, and only for very large temperature gradients. We present results for $L_{\|} / L_{T}=1600$, with the fastest growing wavenumber $k_{\perp 0}=4 \pi / L_{\perp} \approx k_{\perp \max } / 30$ setting the energy-injection scale. While this arrangement does not inject free energy in a realistic fashion, we are able to study the key features of its transfer and dissipation in drift-kinetic turbulence.

While we solve fully spectrally, it is convenient for presentation to write equations in a form spectral in velocity and the parallel spatial direction only. Equation (1) becomes

$$
\begin{aligned}
& \frac{\partial \hat{g}_{m}}{\partial t}+i k_{\|} v_{\mathrm{th}}\left(\sqrt{\frac{m+1}{2}} \hat{g}_{m+1}+\sqrt{\frac{m}{2}} \hat{g}_{m-1}\right) \\
& +\frac{i k_{\|} v_{\mathrm{th}}}{\sqrt{2}} \hat{\varphi} \delta_{m 1}+\sum_{p_{\|}+q_{\|}=k_{\|}} \hat{\mathrm{u}}_{\perp}\left(p_{\|}\right) \cdot \nabla_{\perp} \hat{g}_{m}\left(q_{\|}\right) \\
& =-\nu m^{n} \hat{g}_{m} \mathcal{I}_{m \geq 3}+\hat{\chi},
\end{aligned}
$$

where hats denote functions in $\left(\boldsymbol{r}_{\perp}, k_{\|}, m\right)$ space, and $\hat{\chi}=-\left(\rho_{i} v_{\text {th }} / 2 \sqrt{2} L_{T}\right)(\partial \hat{\varphi} / \partial y) \hat{\varkappa} \delta_{m 2}$. Equation (5) exhibits the two mixing mechanisms present in the turbulence: the "fluid" cascade due to the $\hat{\boldsymbol{u}}_{\perp} \cdot \nabla_{\perp} \hat{g}_{m}$ nonlinearity, and linear phase mixing by coupling with Hermite modes $m \pm 1$.

We first formulate the free-energy transfer between fluid and kinetic moments. Equation (5) implies

$$
\begin{aligned}
& \frac{\partial}{\partial t} \frac{\left|\hat{g}_{m}\right|^{2}}{2}+\Gamma_{m}-\Gamma_{m-1}+\operatorname{Im}\left(\frac{k_{\|} v_{\text {th }}}{\sqrt{2}} \hat{g}_{m} \hat{\varphi}^{*} \delta_{m 1}\right)+N_{m} \\
& \quad=-\nu m^{n}\left|\hat{g}_{m}\right|^{2} \mathcal{I}_{m \geq 3}+\operatorname{Re}\left(\hat{g}_{m}^{*} \hat{\chi}\right)
\end{aligned}
$$

where $N_{m}=\operatorname{Re}\left[\sum_{p_{\|}+q_{\|}=k_{\|}} \hat{g}_{m}\left(k_{\|}\right)^{*} \hat{\boldsymbol{u}}_{\perp}\left(p_{\|}\right) \cdot \nabla_{\perp} \hat{g}_{m}\left(q_{\|}\right)\right]$is the free-energy transfer in $\left(\boldsymbol{r}_{\perp}, k_{\|}\right)$space due to the nonlinearity, and $\Gamma_{m}=k_{\|} v_{\text {th }} \sqrt{(m+1) / 2} \operatorname{Im}\left(\hat{g}_{m+1}^{*} \hat{g}_{m}\right)$ is the free-energy transfer from Hermite mode $m$ to $m+1$ due to phase mixing. ${ }^{29}$ Since we focus on this transfer between Hermite modes $m$, rather than the nonlinear cascade in $\boldsymbol{k}$, we omit the $m$-independent hyperviscous dissipation at large $|\boldsymbol{k}|$. A full theoretical treatment that includes both transfers may be found in Ref. 12 .

Let $W=\int \mathrm{d}^{2} \boldsymbol{r}_{\perp} \sum_{k \|}\left(W_{\text {fluid }}+W_{\text {kin }}\right)$, where $W_{\text {fluid }}\left(\boldsymbol{r}_{\perp}, k_{\|}\right)$ $=(1+\alpha)|\hat{\varphi}|^{2} / 2 \alpha^{2}+\left|\hat{u}_{\|}\right|^{2} / v_{\text {th }}^{2}+\left|\delta \hat{T}_{\|}\right|^{2} / 4 T^{2}$ is the free energy in fluid moments, and $W_{\text {kin }}\left(\boldsymbol{r}_{\perp}, k_{\|}\right)=\sum_{m=3}^{\infty}\left|\hat{g}_{m}\right|^{2} / 2$ is the free energy in kinetic moments. Summing (6) separately over $m \in\{0,1,2\}$ (fluid) and $m \geq 3$ (kinetic) gives

$$
\frac{\partial W_{\text {fluid }}}{\partial t}=\mathcal{M}+\mathcal{S}-\mathcal{T}, \quad \frac{\partial W_{\text {kin }}}{\partial t}=\mathcal{N}+\mathcal{T}-\mathcal{C},
$$


where $\mathcal{S}\left(\boldsymbol{r}_{\perp}, k_{\|}\right)=\operatorname{Re}\left(\delta \hat{T}_{\|}^{*} \hat{\boldsymbol{u}}_{x}\right) /\left(2 T_{i} L_{T}\right)$ is the source due to the ITG, $\mathcal{C}\left(\boldsymbol{r}_{\perp}, k_{\|}\right)=\nu \sum_{m=3}^{\infty} m^{n}\left|\hat{g}_{m}\right|^{2}$ is the sink due to collisions, $\mathcal{M}\left(\boldsymbol{r}_{\perp}, k_{\|}\right)=-\left[(1+\alpha) N_{0}+N_{1}+N_{2}\right]$ and $\mathcal{N}\left(\boldsymbol{r}_{\perp}, k_{\|}\right)$ $=-\sum_{m=3}^{\infty} N_{m}$ are free-energy transfers due to the nonlinearity, and $\mathcal{T}\left(\boldsymbol{r}_{\perp}, k_{\|}\right)=k_{\|} v_{\text {th }} \sqrt{3} \operatorname{Im}\left(\hat{g}_{3}^{*} \delta \hat{T}_{\|}\right) /\left(2 T_{i}\right)=\Gamma_{2}$ is the transfer of free energy from fluid to kinetic moments by the streaming term $v_{\|} \nabla_{\|} g$ in (1). Streaming is linear and reversible, so $\mathcal{T}$ may be positive or negative; however, setting $\mathcal{T}$ $=\mathcal{T}_{L} \equiv\left|k_{\|}\right| v_{\text {th }} \sqrt{3 / 2}\left|\hat{g}_{2}\right|^{2}$ (more generally, $\Gamma_{m}=\Gamma_{m}^{L} \equiv\left|k_{\|}\right| v_{\text {th }}$ $\left.\sqrt{(m+1) / 2}\left|\hat{g}_{m}\right|^{2}\right)$ amounts to an effective Landau-fluidstyle closure that captures free-energy dissipation by Landau damping. While not exact, ${ }^{10}$ it yields spectra in excellent agreement with linear drift-kinetic simulations. ${ }^{25}$

We now consider the saturated state of the turbulence, in which the time averages $\left\langle\mathrm{d} W_{\text {fluid }} / \mathrm{d} t\right\rangle=\left\langle\mathrm{d} W_{\text {kin }} / \mathrm{d} t\right\rangle=0$, so $\langle\mathcal{N}\rangle+\langle\mathcal{T}\rangle=\langle\mathcal{C}\rangle \geq 0$. For linear phase mixing (and Landau damping) to play a similar role in a turbulent plasma as in a linear plasma, $\mathcal{T}$ would have to be similar to its linear value, $\langle\mathcal{T}\rangle \sim \mathcal{T}_{L}$. In Fig. 1, we plot the ratio $\left\langle\mathcal{T} / \mathcal{T}_{L}\right\rangle$ as a function of $\left(k_{\perp}, k_{\|}\right)$(it is isotropic in $\left.\boldsymbol{r}_{\perp}\right)$ for a time average taken over a time window of length $2 L_{\|} / v_{\text {th }}$ in saturated turbulence. The transfer is almost completely suppressed compared to the linear case, $\left\langle\mathcal{T} / \mathcal{T}_{L}\right\rangle \ll 1$, across a large range of wavenumbers that we will shortly characterize.

The suppression of free-energy transfer associated with phase mixing is a nonlinear, kinetic effect. To understand the suppression mechanism, we decompose the distribution function into propagating modes in Hermite space, by writing $\hat{g}_{m}=\left(-i \operatorname{sgn} k_{\|}\right)^{m}\left[\hat{g}_{m}^{+}+(-1)^{m} \hat{g}_{m}^{-}\right]$, where $\hat{g}_{m}^{ \pm}=\frac{1}{2}\left( \pm i \operatorname{sgn} k_{\|}\right)^{m}$ $\left[\hat{g}_{m} \pm i \operatorname{sgn}\left(k_{\|}\right) \hat{g}_{m+1}\right]$. The "phase-mixing mode," $\hat{g}_{m}^{+}$, propagates forward from low to high $m$, the "anti-phase-mixing mode," $\hat{g}_{m}^{-}$, propagates backward from high to low $m .^{10,12,26}$ For $m \geq 3,\left|\hat{g}_{m}^{ \pm}\right|^{2}$ evolves according to

$$
\begin{aligned}
\frac{\partial}{\partial t} \frac{\left|\hat{g}_{m}^{ \pm}\right|^{2}}{2} \pm \frac{\left|k_{\|}\right| v_{\text {th }}}{\sqrt{2}} \frac{\partial}{\partial m}\left(\sqrt{m}\left|\hat{g}_{m}^{ \pm}\right|^{2}\right)+\nu m^{n}\left|\hat{g}_{m}^{ \pm}\right|^{2} \\
=-\operatorname{Re}\left\{\sum_{p_{\|}+q_{\|}=k_{\|}}\left[\hat{g}_{m}^{ \pm}\left(k_{\|}\right)\right]^{*}\right. \\
\left.\quad \times \hat{\boldsymbol{u}}_{\perp}\left(p_{\|}\right) \cdot \nabla_{\perp}\left[\delta_{k_{\|} q_{\|}}^{+} \hat{g}_{m}^{ \pm}\left(q_{\|}\right)+\delta_{k_{\|} q_{\|}}^{-} \hat{g}_{m}^{\mp}\left(q_{\|}\right)\right]\right\},
\end{aligned}
$$

where $\delta_{k_{\|} q_{\|}}^{ \pm}=\frac{1}{2}\left[1 \pm \operatorname{sgn}\left(k_{\|} q_{\|}\right)\right]$so $\delta_{k_{\|} q_{\|}}^{+}$picks out $k_{\|}$and $q_{\|}$ that have the same sign, and $\delta_{k_{\|} q_{\|}}^{-}$the opposite sign. We have introduced a continuous approximation for the finite difference in $m$, valid because $\hat{g}_{m}^{ \pm}$are smooth in the sense that $\hat{g}_{m}^{ \pm} \approx \hat{g}_{m+1}^{ \pm}$to the lowest order at large $m .^{12}$ In terms of $\hat{g}_{m}^{ \pm}$, the normalized free-energy transfer from $m$ to $m+1$ is

$$
\begin{aligned}
\bar{\Gamma}_{m} & \equiv \frac{\Gamma_{m}}{\Gamma_{m}^{L}}=\frac{k_{\|} v_{\text {th }} \sqrt{(m+1) / 2} \operatorname{Im}\left(\hat{g}_{m+1}^{*} \hat{g}_{m}\right)}{k_{\|} v_{\text {th }} \sqrt{(m+1) / 2}\left|\hat{g}_{m}\right|^{2}} \\
& \approx \frac{\left|\hat{g}_{m}^{+}\right|^{2}-\left|\hat{g}_{m}^{-}\right|^{2}}{\left|\hat{g}_{m}^{+}\right|^{2}+\left|\hat{g}_{m}^{-}\right|^{2}},
\end{aligned}
$$

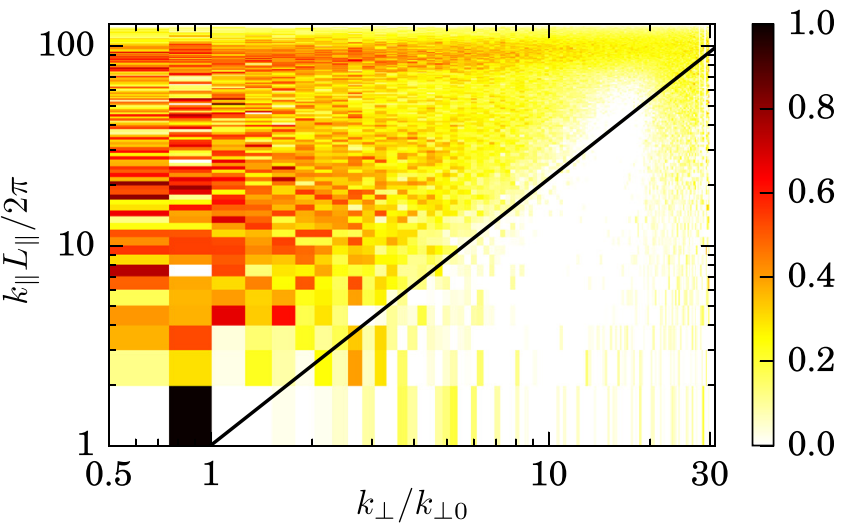

FIG. 1. $\left\langle\bar{\Gamma}_{2}\right\rangle=\left\langle\mathcal{T} / \mathcal{T}_{L}\right\rangle$, the free-energy transfer from fluid to kinetic moments in the saturated turbulent state, normalized to its value in a linear plasma, expressed in Fourier space. We also show the line of critical balance $\tau_{\mathrm{nl}}=\tau_{\mathrm{s}}$, as defined in the text.

since $\left|\hat{g}_{m}\right|^{2} \approx\left|\hat{g}_{m}^{+}\right|^{2}+\left|\hat{g}_{m}^{-}\right|^{2}$ for large $m .^{12}$ Any suppression of free-energy transfer, $\bar{\Gamma}_{m}<1$, can only be due to the antiphase-mixing modes, $\hat{g}_{m}^{-} \neq 0$.

Let us now consider the linear and nonlinear regimes in terms of $\hat{g}_{m}^{ \pm}$. In the linear case, we neglect the right-hand side of (8). Taking an initial disturbance in the fluid moments (low $m$ ) that propagates forwards only, we seek steady solutions with $\hat{g}_{m}^{-}=0$ (any $\hat{g}_{m}^{-}$present in the initial conditions reflects off the zero flux boundary condition at $m=0$ and becomes forward propagating within one streaming time). The steady solutions of (8) are

$$
\begin{aligned}
\left|\hat{g}_{m}^{+}\right|^{2} & =\frac{A\left(k_{\|}\right)}{\sqrt{m}} \exp \left[\left(-m / m_{c}\right)^{n+\frac{1}{2}}\right], \\
m_{c} & =\left[\frac{(n+1 / 2)\left|k_{\|}\right| v_{\mathrm{th}}}{\sqrt{2} \nu}\right]^{1 /(n+1 / 2)},
\end{aligned}
$$

where $A\left(k_{\|}\right)$is an arbitrary function of $k_{\|}$, and $m_{c}$ is the collisional cutoff. ${ }^{9,10}$ For $m \ll m_{c},\left|\hat{g}_{m}^{+}\right|^{2}$ has a $m^{-1 / 2}$ spectrum, while for $m \geq m_{c},\left|\hat{g}_{m}^{+}\right|^{2}$ decays exponentially. As $\hat{g}_{m}^{-}=0$, the normalized Hermite flux (9) is $\bar{\Gamma}_{m}=1$.

To return to nonlinear drift kinetics, we reinstate the nonlinear term in (8). Now, even if $\hat{g}_{m}^{-}$is zero initially, the nonlinear term acts as a source in the "-" equation. This puts some free energy into the $\hat{g}_{m}^{-}$modes, with the result that $\bar{\Gamma}_{m}<1$, as in Fig. 1 . The effect is even clearer at larger intermediate $m$, away from driving and dissipation: see Fig. 2, where we plot $\bar{\Gamma}_{30}$. There are clear regions in wavenumber space where free-energy transfer is as in the linear case, $\bar{\Gamma}_{30} \approx 1$, and regions where free-energy transfer is completely suppressed, $\bar{\Gamma}_{30} \ll 1$.

To understand this partition of wavenumber space, we compare the characteristic rates of the phase mixing and nonlinearity, respectively, through the streaming rate $\tau_{\mathrm{s}}^{-1}$ $\sim k_{\|} v_{\text {th }}$ and the eddy turnover rate, ${ }^{11} \tau_{\mathrm{nl}}^{-1} \sim k_{\perp} u_{\perp} \sim\left(v_{\text {th }} / L_{\|}\right)$ $\left(k_{\perp} / k_{\perp 0}\right)^{4 / 3}$. When $\tau_{\mathrm{s}} \ll \tau_{\mathrm{nl}}$, streaming dominates nonlinearity and the problem is essentially linear with $\bar{\Gamma}_{m}=1$. When $\tau_{\mathrm{nl}} \ll \tau_{\mathrm{s}}$, nonlinearity dominates streaming and $\bar{\Gamma}_{m}<1$. As shown in Figs. 1 and 2, the line of critical balance, $\tau_{\mathrm{nl}}=\tau_{\mathrm{s}}$, is in good agreement with the boundary of complete 


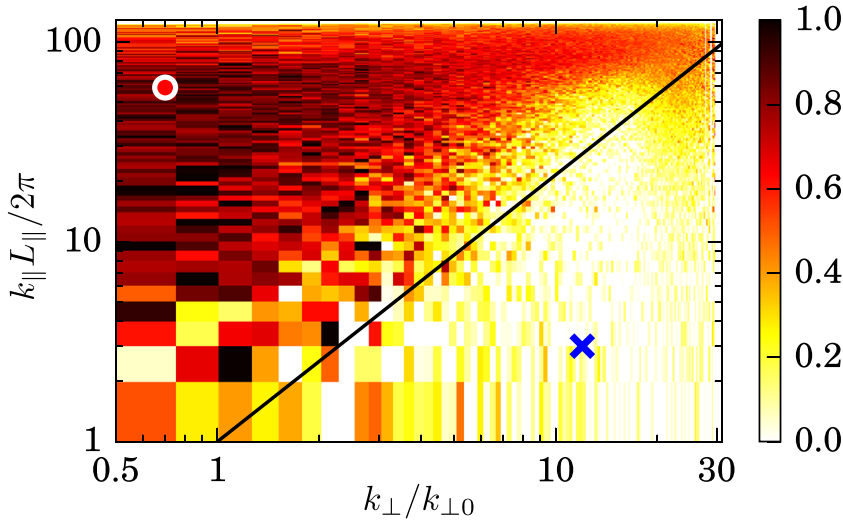

FIG. 2. $\left\langle\bar{\Gamma}_{30}\right\rangle=\left\langle\Gamma_{30} / \Gamma_{30}^{L}\right\rangle$, free-energy transfer from mode 30 to mode 31 , normalized to its value in a linear plasma, expressed in Fourier space. We also show the line of critical balance $\tau_{\mathrm{nl}}=\tau_{\mathrm{s}}$, as defined in the text. The cross and circle mark the locations of the Hermite spectra shown in Fig. 3.

suppression $\bar{\Gamma}_{m}=0$, indicating that free-energy transfer is suppressed whenever $\tau_{\mathrm{nl}} \lesssim \tau_{\mathrm{s}}$.

The scales for which $\tau_{\mathrm{nl}} \lesssim \tau_{\mathrm{s}}$ contain the majority of the free energy, as seen in the theoretically predicted ${ }^{12}$ and numerically measured ${ }^{25}$ spectra. This has two important consequences. First, each velocity scale $m$ is, statistically, very nearly energetically decoupled from other scales, so, in particular, the fluid and kinetic moments are decoupled. Second, since $\sum_{k_{\|}} \int \mathrm{d}^{2} \boldsymbol{r}_{\perp}\langle\mathcal{N}\rangle=0$, the time average of (7) implies that the collisional dissipation rate, $\sum_{k_{\|}} \int \mathrm{d}^{2} \boldsymbol{r}_{\perp}\langle\mathcal{C}\rangle$ $=\sum_{k_{\|}} \int \mathrm{d}^{2} \boldsymbol{r}_{\perp}\langle\mathcal{T}\rangle$, is also strongly suppressed, so collisional dissipation at fine velocity-space scales is a far less effective dissipation channel than in the case of linear Landau damping.

We now confirm this suppression of collisional dissipation by considering the Hermite spectra. The different freeenergy transfer behaviors in the phase-mixing-dominated and the nonlinearity-dominated regions give rise to two different Hermite spectra, plotted in Fig. 3 for fixed wavenumbers and averaged over the same time window of length $2 L_{\|} / v_{\text {th }}$ used in Figs. 1 and 2 . In the phase-mixing-dominated region $\left(\Gamma_{m}=1\right)$, we observe the linear $m^{-1 / 2}$ spectrum given

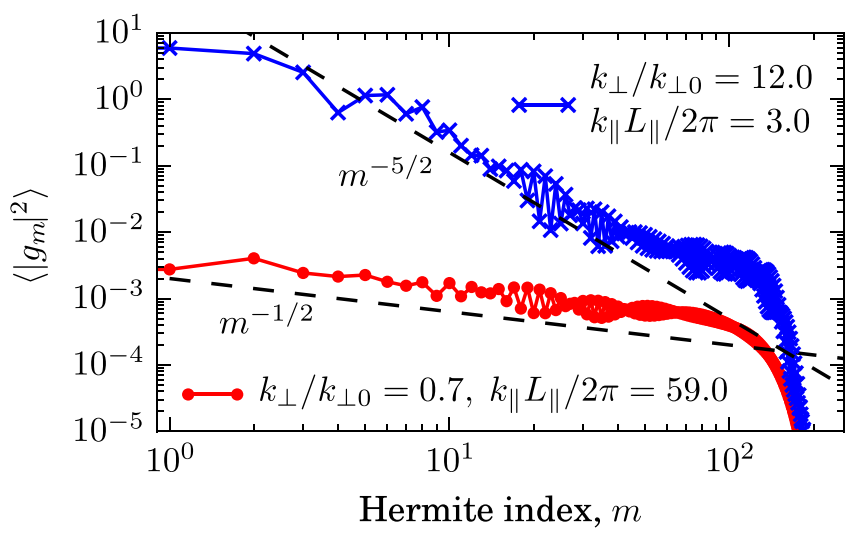

FIG. 3. Time-averaged Hermite spectra for fixed $k_{\|}$and $k_{\perp}$ in the linearstreaming-dominated $\left(\mathrm{m}^{-1 / 2}\right)$ and nonlinearity-dominated $\left(\mathrm{m}^{-5 / 2}\right)$ regions. The $(-1)^{m}$ zig-zag behavior in $\left|\hat{g}_{m}\right|^{2}$ is characteristic of a superposition of both $\hat{g}_{m}^{+}$and $\hat{g}_{m}^{-}$modes. in (10) for $m \ll m_{c}$. In the nonlinearity-dominated region $\left(\Gamma_{m} \ll 1\right)$, we observe the steep $m^{-5 / 2}$ spectrum predicted by Schekochihin et al. ${ }^{12}$ Both spectra exhibit a $(-1)^{m}$ zig-zag behavior that arises from a superposition of both $\hat{g}_{m}^{+}$and $\hat{g}_{m}^{-}$ modes in $\left|\hat{g}_{m}\right|^{2}=\left|\hat{g}_{m}^{+}\right|^{2}+\left|\hat{g}_{m}^{-}\right|^{2}+2(-1)^{m} \operatorname{Re}\left(\hat{g}_{m}^{+*} \hat{g}_{m}^{-}\right)$. The spectrum in the phase-mixing region gives rise to freeenergy dissipation at the usual Landau damping rate: for a fixed Fourier mode in this region, $\int_{3}^{m_{c}} \mathrm{~d} m \nu m m^{-1 / 2} \sim\left|k_{\|}\right| v_{\text {th }}$. This remains finite as $\nu \rightarrow 0^{+}$. In contrast, the dissipation rate for the $m^{-5 / 2}$ spectrum observed in the nonlinear region is $\int_{3}^{m_{c}} \mathrm{~d} m \nu m m^{-5 / 2} \sim \nu^{4 / 3}\left(\left|k_{\|}\right| v_{\mathrm{th}}\right)^{-1 / 3} \rightarrow 0$ as $\nu \rightarrow 0^{+}$. Landau damping is thus suppressed in the nonlinear region. As most of the free energy is contained in this region, ${ }^{12,25}$ the total dissipation via phase mixing to collisional scales in $v_{\|}$tends to zero as $\nu \rightarrow 0^{+}$. The vast majority of free energy cascades nonlinearly to dissipate at fine physical-space scales instead.

These spectra and dissipation patterns explain other numerical observations ${ }^{19,20}$ that the Hermite spectrum summed over all Fourier modes is much steeper than the linear $m^{-1 / 2}$ spectrum, and that free-energy dissipation via collisions in the inertial range decreases as $\nu$ decreases. Those observations may be understood as the result of aggregating the behaviors of the two distinct regions of Fourier space identified above. It is possible to prove ${ }^{12}$ that the aggregate Hermite spectrum is $m^{-2}$, consistent with the reported numerical scalings. ${ }^{19,25}$

In this letter, we have shown that linear phase mixing and the nonlinear cascade are strongly interdependent. The nonlinear cascade in the inertial range excites anti-phasemixing modes, suppressing the net transfer of free energy into kinetic modes. This has both theoretical and practical implications. Theoretically, these results profoundly change our understanding of the way in which free energy is cascaded and dissipated in phase space. As there is only a small net free-energy flux out of fluid modes in the inertial range, it is probably legitimate to neglect parallel phase mixing when deducing physical-space spectra from Kolmogorov arguments, as was done in earlier work. ${ }^{11}$ Since the Hermite spectrum at energetically dominant scales is a steep $m^{-5 / 2}$ power law, these scales experience no free-energy dissipation via Landau damping as $\nu \rightarrow 0^{+}$, and almost all free energy cascades to perpendicular spatial sub-Larmor scales. The steep Hermite spectrum also means that free-energy dissipation via linear phase mixing is not independent of collision frequency. This has the important practical implication that enlarged collision frequencies cannot necessarily be used to compensate for low $v_{\|}$resolution in weakly collisional simulations.

In this work, we have studied electrostatic ITG driftkinetic turbulence. However, our focus is on inertial-range physics, which does not depend on the details of the energy injection. The approach presented here should be applicable to other kinetic systems, where a nonlinearity coexists with particle streaming. Indeed, similar suppressions of phase mixing have already been observed due to a different nonlinearity in the Vlasov-Poisson system, ${ }^{26}$ and in kinetic passive scalar simulations. ${ }^{30} \mathrm{We}$ also expect this work be relevant in electromagnetic plasmas, though these have the complication 
that particle streaming along the perturbed magnetic field line also contributes to the nonlinear term. This will be a subject of future work.

The authors are grateful for fruitful conversations with I. Abel, M. Barnes, G. Colyer, S. Cowley, M. Fox, G. Hammett, F. Parra, C. Roach, and F. van Wyk, and especially A. Kanekar and W. Dorland. This work was supported by the UK Engineering and Physical Sciences Research Council through a Doctoral Training Grant award to J.T.P. and an Advanced Research Fellowship [Grant No. EP/E054625/1] to P.J.D., with additional support from Award No KUK-C1-01304 made by King Abdullah University of Science and Technology (KAUST). Some of the results of this research were obtained using the PRACE-3IP project (FP7 RI312763), resource FIONN based in Ireland at the DJEI/DES/ SFI/HEA Irish Centre for High-End Computing (ICHEC). This work also made use of the IRIDIS High Performance Computing Facility provided by the Science and Engineering South (SES) Centre for Innovation, the UK HECToR HPC facility [Grant No. EP/H002081/1], the resources of the STFC Hartree Centre, the HELIOS supercomputer (IFERC-CSC), Admori, Japan, and the University of Oxford Advanced Research Computing (ARC) facility. ${ }^{31}$ E.G.H's work has been carried out within the framework of the EUROfusion Consortium and was supported by a EUROfusion fusion researcher fellowship [WP14-FRF-CCFE/Highcock]. The views and opinions expressed herein do not necessarily reflect those of the European Commission. All authors are grateful to the Wolfgang Pauli Institute, Vienna, for its hospitality on several occasions.

${ }^{1}$ J. W. Connor and H. R. Wilson, Plasma Phys. Controlled Fusion 36, 719 (1994).

${ }^{2}$ E. J. Doyle, W. A. Houlberg, Y. Kamada, V. Mukhovatov, T. H. Osborne, A. Polevoi, G. Bateman, J. W. Connor, J. G. Cordey, T. Fujita et al., Nucl. Fusion 47, S18 (2007).

${ }^{3}$ X. Garbet, Y. Idomura, L. Villard, and T. H. Watanabe, Nucl. Fusion 50, 043002 (2010).

${ }^{4}$ G. G. Howes, S. C. Cowley, W. Dorland, G. W. Hammett, E. Quataert, and A. A. Schekochihin, Astrophys. J. 651, 590-614 (2006).

${ }^{5}$ A. A. Schekochihin, S. C. Cowley, W. Dorland, G. W. Hammett, G. G. Howes, E. Quataert, and T. Tatsuno, Astrophys. J. Sup. Series 182, 310-377 (2009).

${ }^{6}$ T. Tatsuno, W. Dorland, A. A. Schekochihin, G. G. Plunk, M. Barnes, S. C. Cowley, and G. G. Howes, Phys. Rev. Lett. 103, 015003 (2009).
${ }^{7}$ G. G. Howes, J. M. TenBarge, W. Dorland, E. Quataert, A. A. Schekochihin, R. Numata, and T. Tatsuno, Phys. Rev. Lett. 107, 035004 (2011).

${ }^{8}$ D. Told, F. Jenko, J. M. TenBarge, G. G. Howes, and G. W. Hammett, Phys. Rev. Lett. 115, 025003 (2015).

${ }^{9}$ A. Zocco and A. A. Schekochihin, Phys. Plasmas 18, 102309 (2011).

${ }^{10}$ A. Kanekar, A. A. Schekochihin, W. Dorland, and N. F. Loureiro, J. Plasma Phys. 81, 305810104 (2015).

${ }^{11}$ M. Barnes, F. I. Parra, and A. A. Schekochihin, Phys. Rev. Lett. 107, 115003 (2011).

${ }^{12}$ A. A. Schekochihin, J. T. Parker, E. G. Highcock, P. J. Dellar, W. Dorland, and G. W. Hammett, J. Plasma Phys. 82, 905820212 (2016).

${ }^{13}$ G. G. Howes, S. C. Cowley, W. Dorland, G. W. Hammett, E. Quataert, and A. A. Schekochihin, J. Geophys. Res. 113, A05103, doi:10.1029/ 2007JA012665 (2008).

${ }^{14}$ J. Podesta, J. Borovsky, and S. Gary, Astrophys. J. 712, 685 (2010).

${ }^{15}$ V. Bratanov, F. Jenko, D. R. Hatch, and M. Wilczek, Phys. Rev. Lett. 111, 075001 (2013).

${ }^{16}$ T. Passot and P. L. Sulem, Astrophys. J. Lett. 812, L37 (2015).

${ }^{17}$ P. L. Sulem, T. Passot, D. Laveder, and D. Borgogno, Astrophys. J. 818, 66 (2016).

${ }^{18}$ T.-H. Watanabe and H. Sugama, Nucl. Fusion 46, 24-32 (2006).

${ }^{19}$ D. R. Hatch, F. Jenko, A. B. Navarro, and V. Bratanov, Phys. Rev. Lett. 111, 175001 (2013).

${ }^{20}$ D. R. Hatch, F. Jenko, V. Bratanov, and A. B. Navarro, J. Plasma Phys. 80, 531-551 (2014).

${ }^{21}$ R. W. Gould, T. M. O'Neil, and J. H. Malmberg, Phys. Rev. Lett. 19, 219-222 (1967).

${ }^{22}$ J. H. Malmberg, C. B. Wharton, R. W. Gould, and T. M. O’Neil, Phys. Rev. Lett. 20, 95 (1968).

${ }^{23}$ This echo effect could not be captured in the earlier work by Plunk, ${ }^{32}$ who modelled the effect of turbulence not as nonlinear advection but as a random force in a linear system. There could thus not be any nonlinear modification of the linear response, so the effective damping rate inferred by Plunk differed from the standard Landau damping rate only because the kinetic Langevin system is functionally different from the standard fluid system. ${ }^{10}$

${ }^{24} \mathrm{We}$ ignore here the subtleties of the $k_{\|}=0$ electron response in ITG turbulence (see, e.g., Ref. 33, sec. J.2) - they do not matter for the inertial-range physics on which we focus here. The response we use is formally correct for electron-temperature-gradient (ETG) turbulence, ${ }^{34}$ which is described by the same equations with $e \leftrightarrow i$ swapped and some irrelevant sign changes (Ref. 12, sec. 2.2.1).

${ }^{25}$ J. T. Parker, D.Phil thesis, University of Oxford (2015) arXiv:1603.04727.

${ }^{26}$ J. T. Parker and P. J. Dellar, J. Plasma Phys. 81, 305810203 (2015).

${ }^{27}$ J. G. Kirkwood, J. Chem. Phys. 14, 180-200 (1946).

${ }^{28}$ A. Lenard and I. B. Bernstein, Phys. Rev. 112, 1456-1459 (1958).

${ }^{29}$ T.-H. Watanabe and H. Sugama, Phys. Plasmas 11, 1476 (2004).

${ }^{30}$ A. V. Kanekar, Ph.D. thesis, University of Maryland, College Park (2014), http://drum.lib.umd.edu/handle/1903/16418.

${ }^{31}$ A. Richards, "University of Oxford advanced research computing," (2015), http://dx.doi.org/10.5281/zenodo.22558.

${ }^{32}$ G. G. Plunk, Phys. Plasmas 20, 032304 (2013).

${ }^{33}$ I. G. Abel and S. C. Cowley, New J. Phys. 15, 023041 (2013).

${ }^{34}$ W. Dorland, F. Jenko, M. Kotschenreuther, and B. N. Rogers, Phys. Rev. Lett. 85, 5579-5582 (2000). 